\title{
Adaptive Super-Twisting Observer for Estimation of Random Road Excitation Profile in Automotive Suspension Systems
}

\author{
J. J. Rath, ${ }^{1}$ K. C. Veluvolu, ${ }^{1}$ and M. Defoort ${ }^{2}$ \\ ${ }^{1}$ School of Electronics Engineering, Kyungpook National University, Daegu 702-701, Republic of Korea \\ ${ }^{2}$ LAMIH, CNRS UMR 8201, University Lille Nord de France, UVHC, 59313 Valenciennes, France
}

Correspondence should be addressed to K. C. Veluvolu; veluvolu@ee.knu.ac.kr

Received 31 August 2013; Accepted 4 December 2013; Published 9 February 2014

Academic Editors: F. Berto and I. Uzmay

Copyright (C) 2014 J. J. Rath et al. This is an open access article distributed under the Creative Commons Attribution License, which permits unrestricted use, distribution, and reproduction in any medium, provided the original work is properly cited.

\begin{abstract}
The estimation of road excitation profile is important for evaluation of vehicle stability and vehicle suspension performance for autonomous vehicle control systems. In this work, the nonlinear dynamics of the active automotive system that is excited by the unknown road excitation profile are considered for modeling. To address the issue of estimation of road profile, we develop an adaptive supertwisting observer for state and unknown road profile estimation. Under Lipschitz conditions for the nonlinear functions, the convergence of the estimation error is proven. Simulation results with Ford Fiesta MK2 demonstrate the effectiveness of the proposed observer for state and unknown input estimation for nonlinear active suspension system.
\end{abstract}

\section{Introduction}

The automotive vehicle suspension dynamics contribute significantly in evaluating the effective performance with regard to passenger comfort, road handling, and stability of the vehicle [1]. The design of suspension systems evolved from passive suspensions to the active suspension system [2] adding more control capabilities. Suspension dynamics predominantly provide information regarding vertical stability $[2,3]$ of the vehicle. Effective analysis of the suspension performance provides information regarding the vertical load acting on the vehicle, a critical component in determining the effective tractive force [3]. In such scenarios, the suspension system dynamics that replicate the behavior of mass spring damper system [3] are complex to analyze when nonlinear behavior of the spring and the damper systems are considered. Road profile that replicates the randomness of road surface in form of cleats or troughs affects the suspension system performance [4]. The different levels of road excitation necessitate the continuous regulation of damping force generated by the suspension for maintaining the stability of the vehicle. As the vehicle operating range varies, analysis of the nonlinear dynamics of the suspension system excited by the road profile is one of the major domains of research for suspension systems.
Road profile is a critical parameter that results in undesirable vertical vibrations for the vehicle if it is not compensated by an adequate control effort in the suspension. These unfavorable vibrations result in dynamic variations in the vertical load of the vehicle affecting its stability. The design of suitable controllers to compensate for these variations is dependent on the effective measurement of the road profile. For experimental purposes, expensive instruments called profilographs are used for measuring the road profile [4, 5]. In [6], a real time conditioning algorithm was designed to measure the road profile based on measurement of the vertical acceleration. As an alternative to these expensive instrumentation and sensor technology that are affected by noise, estimation of the road profile by use of observers has been an important issue.

In [7], a Kalman filter-based estimation of the road profile measured using a road profilometer was performed. The designed estimation worked on the vertical dynamics of an active suspension system and was experimentally validated. In [8], a neural network-based approach was adopted to estimate the random road profile. This work employed accelerometers to estimate the road profile modeled as a function of road roughness coefficient. In [9], a minimum 
order observer was designed for a linearized model of passive suspension dynamics to estimate the road profile.

Sliding mode theory $[10,11]$ has evolved over time as an effective tool for estimation of unknown inputs in control domain. In [12], a first order sliding mode observer was designed to estimate the states of the suspension system under the influence of different road profiles. To remove the chattering effect that is inherent in first order sliding mode [13-17], higher order sliding mode theory was developed. Recently, HOSM observers have been popular for state and unknown input estimation in uncertain nonlinear systems [18-20]. For systems with relative degree one, the supertwisting algorithm (STA) served as an ideal tool for the estimation of unknown inputs [21]. In [22-24], the STA based observer was used to estimate the road profile acting as unknown input to the system. By measurement of the vertical velocities, the road profile and the tire forces were estimated. All these works [7-9] did not consider the nonlinear dynamics of the suspension system. Other works [22-24] did not consider the randomness of the road profile.

To address these issues, we consider the nonlinear dynamics of the active suspension system for a quarter vehicle excited by a random road profile. The suspension dynamics considered in this paper effectively replicate the nonlinear behavior of the spring and damper of the suspension. The road excitation profile is considered as an unknown input in this work and is estimated with an adaptive STA observer [25]. For analysis, the road roughness values are based on power spectral density (PSD) values as proposed by International Organization for Standardization (ISO) [4, 5]. For the design of the observer, under the rank conditions for the output matrix, the system is then partitioned into two subsystems where the unknown input appears in one subsystem. For the subsystem affected by the unknown input, an adaptive STA based observer is then designed to ensure the stability of the error dynamics of the subsystem in finite time. For the subsystem without unknown inputs, a nonlinear observer is designed under Lipschitz conditions to ensure the stability of the system in sliding mode. The application of the proposed method to the modelled vehicle dynamics is validated through simulations.

Throughout this paper, $\lambda_{\max }(\mathbf{A})$ denotes the maximum eigenvalue of matrix $\mathbf{A},\|\mathbf{A}\|$ denotes the 2-norm $\sqrt{\lambda_{\max }\left(\mathbf{A}^{T} \mathbf{A}\right)}$ of a matrix $\mathbf{A}$, and $\sigma_{\text {min }}(\mathbf{A})$ represents the minimum singular value of matrix A. For any vector $z=\left[z_{1}, \ldots, z_{q}\right]^{T} \in R^{q}$ and any scalar $\alpha \in R$, we denote

$$
\begin{gathered}
\operatorname{sign}(z)=\left[\operatorname{sign}\left(z_{1}\right), \ldots, \operatorname{sign}\left(z_{q}\right)\right]^{T}, \\
|z|^{\alpha}=\operatorname{diag}\left(\left|z_{1}\right|^{\alpha}, \ldots,\left|z_{q}\right|^{\alpha}\right), \\
\lceil z\rfloor^{\alpha}=|z|^{\alpha} \operatorname{sign}(z) .
\end{gathered}
$$

\section{Modeling Active Suspension Dynamics}

The active suspension system in vehicles incorporates an active controlled force actuator instead of the shock absorber generally found in a passive suspension system. The nonlinear

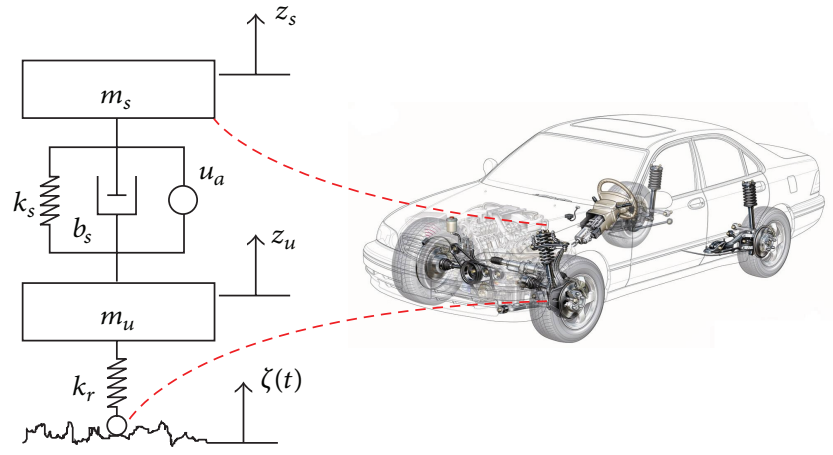

FIGURE 1: Active suspension system for a quarter wheel.

dynamics that govern the active suspension system are given as [26]

$$
\begin{gathered}
m_{s} \ddot{z}_{s}+f_{k}+f_{b}=u, \\
m_{u} \ddot{z}_{u}-f_{k}-f_{b}+k_{r}\left(z_{u}-\zeta(t)\right)=-u,
\end{gathered}
$$

where $m_{s}$ is the sprung mass or the vehicle mass, $m_{u}$ is the unsprung mass or the wheel mass, $z_{s}$ is the sprung mass displacement, $z_{u}$ is the unsprung mass displacement, $u$ is the controlled actuator force, $k_{r}$ is the tire stiffness, and $\zeta(t)$ is the road excitation profile. The nonlinear damping force, $f_{b}$, and spring force, $f_{k}$, for the suspension dynamics can be described as [26]

$$
\begin{gathered}
f_{k}=k_{s}\left(z_{s}-z_{u}\right)+k_{s \mathrm{l} l}\left(z_{s}-z_{u}\right)^{3}, \\
f_{b}=b_{s}\left(\dot{z}_{s}-\dot{z}_{u}\right)+b_{s \mathrm{nl}} \sqrt{\left|\dot{z}_{s}-\dot{z}_{u}\right|} \operatorname{sign}\left(\dot{z}_{s}-\dot{z}_{u}\right),
\end{gathered}
$$

where $k_{s}$ is the linear spring stiffness constant, $b_{s}$ is the linear damper constant, $k_{s n l}$ is the nonlinear spring stiffness, and $b_{s n l}$ is the nonlinear damping constant. The motion of the vehicle over a bump that restricts the wheel travel within a given range and prevents contact between the tyre and the vehicle body is effectively modeled by the nonlinear spring force, $f_{k}$. Similarly for the damper, the damping force generated while the wheel traverses in vertical direction owing to road profile is a nonlinear effect. This nonlinear effect is well approximated by the nonlinear dynamics as depicted in (5). In this work, the damping force provided by the tyre which is very complex to model has been neglected. The active suspension dynamics for a quarter wheel vehicle model together with the modeling parameters are shown in Figure 1. The active suspension dynamics are affected by the road profile requiring control of the effective damping force needed to be provided by the actuator for good handling of the vehicle [1], ride performance, and road stability. Road profile is often modeled as a sinusoidal disturbance or a trapezoidal disturbance to identify with crests/trough or cleats that appear on practical roads. This type of modeling however does not represent the typical roughness profile of roads and the resulting effects it has on the suspension. The standards of road roughness according to ISO $[4,5]$ can be classified into different road classes as shown in Table 1. 
TABLE 1: Road roughness values classified by ISO $[4,5]$.

\begin{tabular}{lcc}
\hline & $\begin{array}{c}\text { Degree of roughness } \sigma_{0} \times 10^{-6} \\
\text { Road class }\end{array}$ & Geometric mean \\
\hline A (very good) & $<8$ & 4 \\
B (good) & $8-32$ & 16 \\
C (average) & $32-128$ & 64 \\
D (poor) & $128-512$ & 256 \\
E (very poor) & $512-2048$ & 1024 \\
F & $2048-8192$ & 4096 \\
\hline
\end{tabular}

The integrated dynamics (2)-(5) for the active suspension system can be represented in state space as

$$
\begin{gathered}
\dot{x}=A x+B \Psi(x, u, t)+E \zeta(t), \\
y=C x .
\end{gathered}
$$

With

$$
\begin{gathered}
x=\left[\begin{array}{cccc}
x_{1} & x_{2} & x_{3} & x_{4}
\end{array}\right]^{T}=\left[\begin{array}{llll}
\dot{z}_{u} & \dot{z}_{s} & z_{s} & z_{u}
\end{array}\right]^{T}, \\
A=\left[\begin{array}{cccc}
-\frac{b_{s}}{m_{u}} & \frac{b_{s}}{m_{u}} & \frac{k_{s}}{m_{u}} & -\frac{k_{s}+k_{r}}{m_{u}} \\
\frac{b_{s}}{m_{s}} & -\frac{b_{s}}{m_{s}} & -\frac{k_{s}}{m_{s}} & \frac{k_{s}}{m_{s}} \\
0 & 1 & 0 & 0 \\
1 & 0 & 0 & 0
\end{array}\right], \quad B=\left[\begin{array}{c}
\frac{1}{m_{u}} \\
-\frac{1}{m_{s}} \\
0 \\
0
\end{array}\right], \\
E=\left[\begin{array}{c}
\frac{k_{r}}{m_{u}} \\
0 \\
0 \\
0
\end{array}\right],
\end{gathered}
$$

where

$$
\begin{aligned}
\Psi(x, u, t)= & -u+k_{\text {snl }}\left(x_{3}-x_{4}\right)^{3} \\
& +b_{\text {snl }} \sqrt{\left|x_{2}-x_{1}\right|} \operatorname{sign}\left(x_{2}-x_{1}\right) .
\end{aligned}
$$

In the modeled system dynamics, the active actuator control force $u$ is the control input for the system. In the system dynamics (6), the displacement of the sprung mass, $x_{3}$, and velocity of the unsprung mass, $x_{1}$, are considered as outputs, and the output matrix can be defined by

$$
C=\left[\begin{array}{llll}
1 & 0 & 0 & 0 \\
0 & 0 & 1 & 0
\end{array}\right] .
$$

The unknown input for the system is the road profile denoted as $\zeta(t)$. The measurement of road profile is an extremely complex task that requires the use of complex measuring instruments such as profilographs $[4,5]$ that are expensive and impractical. Hence our focus is on the development of an approach to estimate the random road profile, $\zeta(t)$, for the active suspension systems.

\section{Observer Design}

In this section, we discuss the design of the observer for the active suspension system. A combination of nonlinear Lipschitz observer and adaptive super-twisting observer is employed. To facilitate the design of the observer, the following assumptions are required.

Assumption 1. All invariant zeros of the triple $(A, E, C)$ must lie in the left half plane and $\operatorname{rank}(C E)=\operatorname{rank}(E)$.

Assumption 2. The nonlinear functions in $\Psi(x, u, t)$ satisfiy the Lipschitz conditions.

Assumption 3. The function $\zeta(t)$ and its first derivative are bounded.

Assumption 4. The control input is bounded and the system is assumed to be bounded input bounded state stable (BIBS).

For the dynamics defined in (6), it can be easily verified that the $\operatorname{rank}(C E)=\operatorname{rank}(E)=1$. Further, the triple $(A, E, C)$ does not contain any invariant zeros that lie on the right hand plane. The nonlinear function $\Psi(x, u, t)$ in the system dynamics (6) can be divided into $\Psi_{1}(x, u, t)$ and $\Psi_{2}(x, u, t)$ to analyze the Lipschitz continuity as follows:

$$
\begin{gathered}
\Psi(x, u, t)=\Psi_{1}(x, u, t)+\Psi_{2}(x, u, t), \\
\Psi_{1}(x, u, t)=k_{\text {snl }}\left(x_{3}-x_{4}\right)^{3}, \\
\Psi_{2}(x, u, t)=b_{\text {snl }} \sqrt{\left|x_{2}-x_{1}\right|} \operatorname{sign}\left(x_{2}-x_{1}\right) .
\end{gathered}
$$

With $x_{3}$, that is, $z_{s}$ and $x_{4}$, that is, $z_{u}$ representing the physical states of practically realizable automotive suspension system, the function $\Psi_{1}(x, u, t)$ can be determined to be locally Lipschitz with a Lipschitz constant obtained as $l_{\Psi_{1}}=$ $k_{s n l}\left|z_{s}-z_{u}\right|^{2}$. Similarly for the nonlinear function $\Psi_{2}(x, u, t)$, with $x_{2}$, that is, $\dot{z}_{s}$ and $x_{1}$, that is, $\dot{z}_{u}$ bounded, local Lipschitz continuity can be established. The Lipschitz constant for $\Psi_{2}(x, u, t)$ is obtained as $l_{\Psi_{2}}=\left(b_{s n l} / 2\right)\left(1 /\left|\dot{z}_{s}-\dot{z}_{u}\right|^{1 / 2}\right)$. For the active automotive suspension system modeled in (6), by use of the actuator control force, $u_{a}$, it can be asserted that $\left(\dot{z}_{s}-\dot{z}_{u}\right) \neq 0$ such that the function $\Psi(x, u, t)$ maintains its Lipschitz continuity with a Lipschitz constant, $l_{\beta}=l_{\Psi_{1}}+l_{\Psi_{2}}$.

In the modeled system dynamics (6), the unknown input $\zeta(t)$ is the road profile that is considered as function of road roughness coefficient and other physical parameters relating to the road conditions which are bounded. It can be thus deduced from the dynamics of the road profile model that the road excitation profile and its derivative are both bounded. For the modeled active suspension dynamics (6), the control input to the system is the actuator force, $u$, that is bounded.

In order to design the combined observer, the original system dynamics will be divided into two subsystems, such that one of the subsystems will be free from unknown inputs. With the Assumptions 1-4 for the active suspension system, 


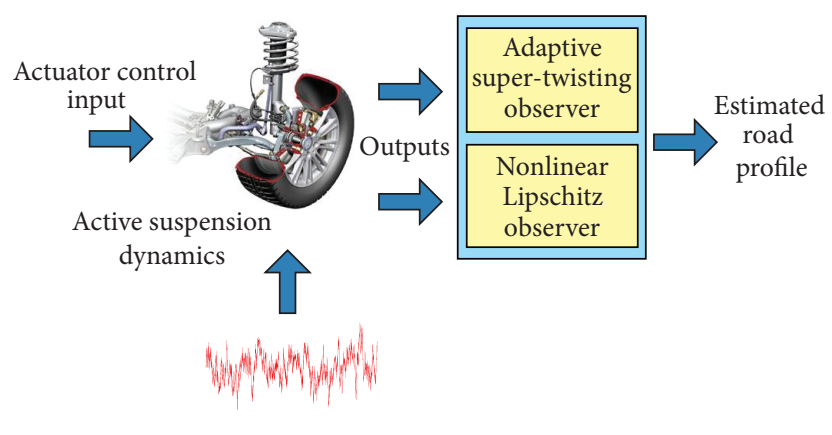

Random road profile

FIGURE 2: Overview of proposed observer.

(6) being satisfied, we can directly partition system (6) into two subsystems $S 1$ and $S 2$ as follows:

$$
\begin{gathered}
S 1:\left\{\begin{array}{l}
\dot{x}_{1}=A_{11} x_{1}+A_{12} z+B_{1} \Psi(x, u, t)+E_{1} \zeta(t), \\
y_{1}=C_{1} x_{1},
\end{array}\right. \\
S 2:\left\{\begin{array}{l}
\dot{x}_{2}=A_{21} x_{1}+A_{22} z+B_{2} \Psi(x, u, t), \\
y_{2}=C_{2} z,
\end{array}\right.
\end{gathered}
$$

where

$$
\begin{aligned}
& x_{1}=x_{1}, \quad z=\left[\begin{array}{lll}
x_{1} & x_{2} & x_{3}
\end{array}\right]^{T}, \\
& C_{1}=[1], \quad C_{2}=\left[\begin{array}{lll}
0 & 1 & 0
\end{array}\right], \\
& A_{11}=\left[-\frac{b_{s}}{m_{u}}\right], \quad A_{12}=\left[\begin{array}{c}
\frac{b_{s}}{m_{u}} \\
\frac{k_{s}}{m_{u}} \\
-\frac{k_{s}+k_{r}}{m_{u}}
\end{array}\right]^{T} \text {, } \\
& A_{21}=\left[\begin{array}{c}
\frac{b_{s}}{m_{s}} \\
0 \\
1
\end{array}\right], \quad A_{22}=\left[\begin{array}{ccc}
-\frac{b_{s}}{m_{s}} & -\frac{k_{s}}{m_{s}} & \frac{k_{s}}{m_{s}} \\
1 & 0 & 0 \\
0 & 0 & 0
\end{array}\right] \text {, } \\
& B_{1}=\left[\frac{1}{m_{u}}\right], \quad B_{2}=\left[\begin{array}{c}
\frac{-1}{m_{s}} \\
0 \\
0
\end{array}\right], \quad E_{1}=\left[\frac{k_{r}}{m_{u}}\right] .
\end{aligned}
$$

With the system (6) partitioned as above, the objective is to design an adaptive STA based observer to estimate the states and unknown input for the $S 1$-subsystem (11) and a nonlinear Lipschitz observer (NLO) to estimate the states for the $S 2$-subsystem (12). The overview of the design is shown in Figure 2.

The estimation error can be defined as

$$
e=\widehat{x}-x=\left[\begin{array}{ll}
e_{1} & e_{z}
\end{array}\right]^{T}=\left[\begin{array}{lll}
e_{1} & {\left[\begin{array}{lll}
e_{2} & e_{3} & e_{4}
\end{array}\right]^{T}}
\end{array}\right]^{T},
$$

where $\widehat{x}$ is the observed state and $e_{1}$ and $e_{z}$ are the errors for the subsystems $S 1$ and $S 2$.

3.1. Adaptive Super-Twisting Observer Design for S1-Subsystem. For S1-subsystem (11) satisfying the above assumptions, the following observer based on the adaptive STA can be designed to estimate the states and the unknown input:

$$
\dot{\hat{x}}_{1}=A_{11} \widehat{x}_{1}+A_{12} \widehat{z}+B_{1} \Psi(\widehat{x}, u, t)+E_{1} v(t),
$$

where $\nu(t)$ is the robust sliding term based on the adaptive STA [25] and defined as

$$
\nu(t)=-K_{1}\left[\widehat{x}_{1}-x_{1}\right\rfloor^{1 / 2}-K_{2} \int_{0}^{t} \operatorname{sign}\left(\widehat{x}_{1}-x_{1}\right) .
$$

The adaptive gains $K_{1}, K_{2}$ in (16) are designed as

$$
\begin{array}{ll}
\dot{K}_{1} & = \begin{cases}\kappa_{1} \operatorname{sign}\left(\left|\widehat{x}_{1}-x_{1}\right|-\epsilon\right), & \text { if } K_{1}>\alpha_{m}, \\
\kappa_{2}, & \text { if } K_{1} \leq \alpha_{m},\end{cases} \\
K_{2} & =\kappa_{3} K_{1},
\end{array}
$$

where $\kappa_{1}, \kappa_{2}, \kappa_{3}$, and $\epsilon$ are positive constants. The parameter $\alpha_{m}$ is an arbitrary small positive constant.

To establish the convergence of the observer dynamics, the error dynamics (14) can be obtained as $e_{1}=\widehat{x}_{1}-x_{1}$, which serves as the sliding surface for the designed adaptive STA based observer. The objective of the designed observer is to ensure that the error converges to zero and to reconstruct the unknown road excitation profile, $\zeta(t)$, from the robust term (16).

Theorem 5. For system (11) satisfying the Assumptions 1-4, the observer system (15) with the robust term (16) will ensure that the error dynamics $\left(e_{1}\right)$ will converge to zero in finite time.

Proof. The error dynamics of the system (11) can be obtained from (11) and (15) as

$$
\begin{aligned}
\dot{e}_{1}= & A_{11} e_{1}+A_{12} e_{z}+B_{1} \Psi(\widehat{x}, u, t)-B_{1} \Psi(x, u, t) \\
& +v(t)-E_{1} \zeta(t) \\
= & \Lambda\left(e_{1}, e_{z}, t\right)+\nu(t)
\end{aligned}
$$

where $\Lambda\left(e_{1}, e_{z}, t\right)$ includes the perturbation terms. The matrices $A_{11}$ and $A_{12}$ are known, and hence they are bounded. As system satisfies Assumptions 2-4, the boundedness of the nonlinear function $\Psi(\widehat{x}, u, t)$ and the unknown input, $\zeta(t)$, can be easily established. For the obtained error dynamics (18), it can be proved that $\dot{e}_{1}$ is locally bounded by a constant, as $e_{1}$ is twice differentiable on a compact set. This is not restrictive as the active suspension system dynamics are bounded at least locally. It will be shown later that the subsystem $S 2$ is asymptotically stable. Further, as the system is free from unknown inputs, under the Assumptions 2-4, the subsystem $S 2$ boundedness can be established. Based on the above arguments, the boundedness of the perturbation $\Lambda\left(e_{1}, e_{z}, t\right)$ is obtained as

$$
\dot{\Lambda}\left(e_{1}, e_{z}, t\right) \leq \rho,
$$


where $\rho$ is a constant (not necessarily known). With the perturbation terms $\Lambda\left(e_{1}, e_{z}, t\right)$, satisfying the condition (19) required for the adaptive STA (15), the convergence of the error dynamics (18) can now be proved with the following Lyapunov function:

$$
V\left(e_{1}\right)=\Omega^{T} P \Omega+\frac{1}{2 \tau_{1}}\left(K_{1}-K_{1}^{*}\right)^{2}+\frac{1}{2 \tau_{2}}\left(K_{2}-K_{2}^{*}\right)^{2},
$$

where $\Omega=\left[\left|e_{1}\right|^{1 / 2} \operatorname{sign}\left(e_{1}\right) e_{1}\right]^{T}$ and $\tau_{1}, \tau_{2}, K_{1}^{*}$, and $K_{2}^{*}$ are positive constants and $P$ is a positive definite matrix. Similar to the results in [25] with $K_{1}$ and $K_{2}$ satisfying (17), $\dot{V}\left(e_{1}\right)$ can be shown to be a negative definite and the error converges to zero in finite time. The sliding surface is thus reached in finite time and maintained thereafter.

3.2. Nonlinear Lipschitz Observer for S2-Subsystem. For subsystem (12), a NLO is designed as follows to estimate the states of the system:

$$
\dot{\widehat{z}}=A_{21} x_{1}+A_{22} \widehat{z}+B_{2} \Psi(\widehat{x}, u, t)+L\left(y_{2}-C_{2} z\right),
$$

where the feedback $L=\left[\begin{array}{lll}l_{11} & l_{21} & l_{31}\end{array}\right]^{T}$ is to be discussed in Theorem 6 later. The error dynamics (14) of the subsystem S2 can be obtained as

$$
\begin{aligned}
{\left[\begin{array}{c}
\dot{e}_{2} \\
\dot{e}_{3} \\
\dot{e}_{4}
\end{array}\right]=} & {\left[\begin{array}{ccc}
-\frac{b_{s}}{m_{s}} & -\frac{k_{s}}{m_{s}}-l_{11} & \frac{k_{s}}{m_{s}} \\
1 & -l_{21} & 0 \\
0 & -l_{31} & 0
\end{array}\right] } \\
& +\left[\begin{array}{c}
-1 \\
0 \\
0
\end{array}\right](\Psi(\widehat{x}, u, t)-\Psi(x, u, t)) .
\end{aligned}
$$

The following theorem establishes the stability of the $S 2$ subsystem.

Theorem 6. For system (12) satisfying the Assumptions 1-4, the observer (21) ensures that the state estimation error $\left(e_{z}\right)$ is asymptotically stable provided that the gain L satisfies

$$
Q\left(A_{22}-L C_{2}\right)+\left(A_{22}-L C_{2}\right)^{T} Q+l_{\beta}^{2} Q Q+I<0,
$$

where $l_{\beta}$ is the Lipschitz constant for $\Psi(x, u, t)$ (Assumption 2) and $Q$ is a positive definite matrix.

Proof. With the convergence of the subsystem $S 1$ error $\left(e_{1}\right)$ to zero in the sliding mode, the error dynamics (14) can be written as

$$
\dot{e}_{z}=\left(A_{22}-L C_{2}\right)+(\Psi(\widehat{x}, u, t)-\Psi(x, u, t)) .
$$

With the system satisfying Assumption 2, the Lipschitz constant for $\Psi(x, u, t)$ is evaluated as $l_{\beta}$. With the choice of the Lyapunov function as $V\left(e_{z}\right)=e_{z}^{T} Q e_{z}$ and differentiating with respect to time, one has

$$
\begin{aligned}
\dot{V}\left(e_{z}\right)= & e_{z}^{T}\left[\left(A_{22}-L C_{2}\right)^{T} Q+Q\left(A_{22}-L C_{2}\right)\right] e_{z} \\
& +2 e_{z}^{T} Q(\Psi(\widehat{x}, t)-\Psi(x, t)) .
\end{aligned}
$$

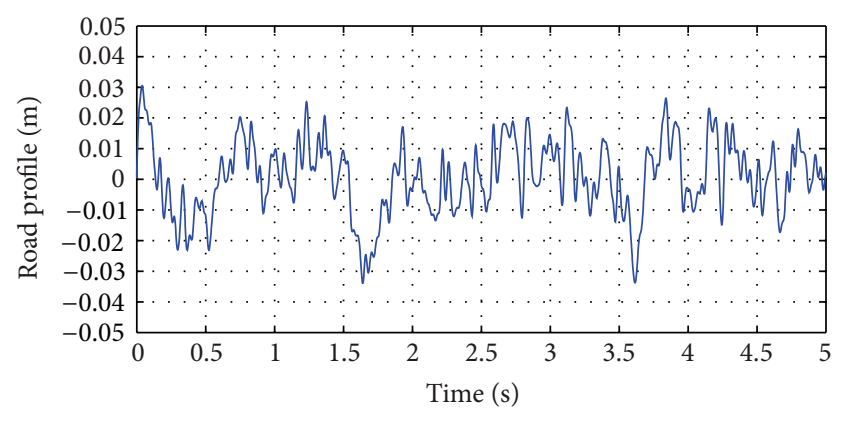

Figure 3: Average Class C road profile.

From the above results, its can be deduced that

$$
\begin{aligned}
\dot{V}\left(e_{z}\right) \leq & e_{z}^{T}\left[\left(A_{22}-L C_{2}\right)^{T} Q+Q\left(A_{22}-L C_{2}\right)\right] e_{z} \\
+ & 2 l_{\beta}\|Q e\|\left\|e_{z}\right\| .
\end{aligned}
$$

In the sliding mode as $e_{1}=0$, we have

$$
e=\left[\begin{array}{ll}
0 & e_{z}
\end{array}\right]^{T}=\left[\begin{array}{llll}
0 & {\left[\begin{array}{lll}
e_{2} & e_{3} & e_{4}
\end{array}\right]^{T}}
\end{array}\right]^{T} .
$$

It can thus be written as follows:

$$
\begin{aligned}
\dot{V}\left(e_{z}\right) \leq & e_{z}^{T}\left[\left(A_{22}-L C_{2}\right)^{T} Q+Q\left(A_{22}-L C_{2}\right)\right] e_{z} \\
& +2 l_{\beta}\left\|Q e_{z}\right\|\left\|e_{z}\right\| .
\end{aligned}
$$

Further, one can obtain

$$
\begin{aligned}
\dot{V}\left(e_{z}\right) \leq & e_{z}\left(\left(A_{22}-L C_{2}\right)^{T} Q+Q\left(A_{22}-L C_{2}\right)+l_{\beta}^{2} Q Q+I\right) \\
& \times e_{z},
\end{aligned}
$$

where $2 l_{\beta}\left\|Q e_{z}\right\|\left\|e_{z}\right\| \leq\left(l_{\beta}\right)^{2} e_{z} Q Q e_{z}+e_{z}^{T} e_{z}$ is satisfied. If the design of the feedback gain, $L$, is such that (23) is satisfied, then $\dot{V}\left(e_{2}\right)<0$. The error dynamics will thus be asymptotically stable.

Remark 7. Equation (23) can be written as an algebraic Riccati equation in the following form:

$$
Q\left(A_{22}-L C_{2}\right)+\left(A_{22}-L C_{2}\right)^{T} Q+l_{\beta}^{2} Q Q+I+\gamma I=0
$$

for some $\gamma>0$. The following condition $[27,28]$ ensures the asymptotic stability of the system (24):

$$
\min _{w \in \mathbb{R}^{+}} \sigma_{\min }\left(A_{22}-L C_{2}-j w I\right)>l_{\beta},
$$

where $\sigma_{\min }(\cdot)$ represents the minimum singular value of a matrix. If the above condition (23) is satisfied and if there exists a stable $\left(A_{22}-L C_{2}\right)$ matrix, then there exists a symmetric positive definite (SPD) solution $Q=Q^{T}$ for the Riccati equation (30). 


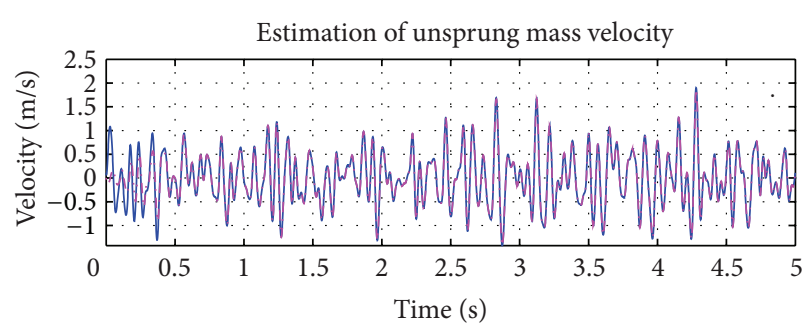

$-\dot{z}_{u}$

(a)

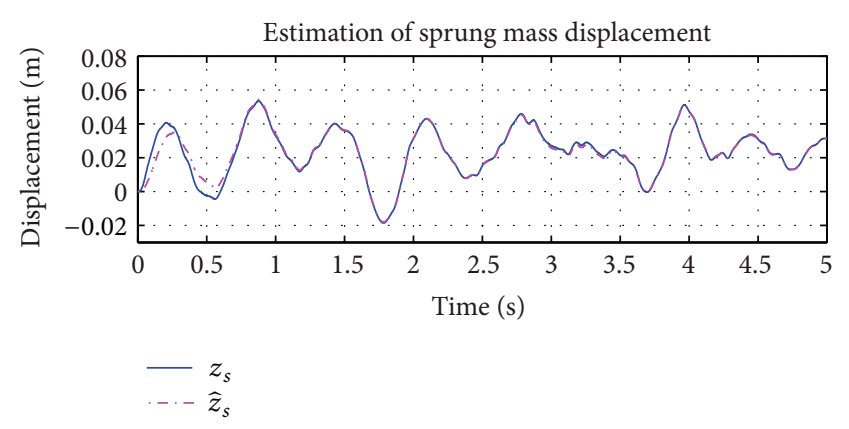

(c)

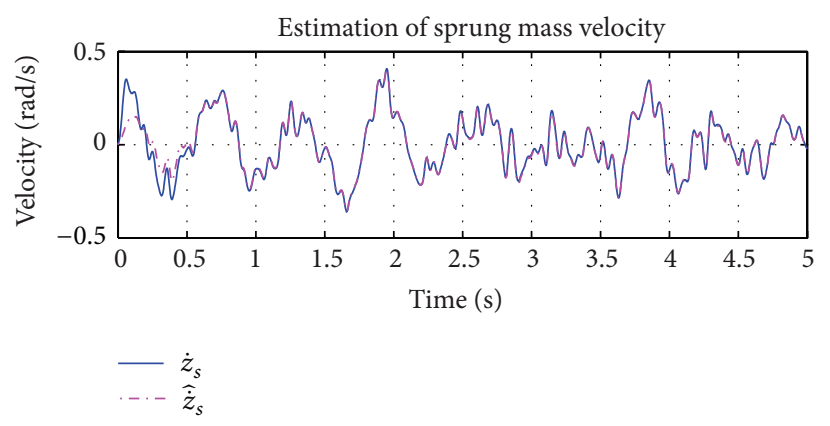

(b)

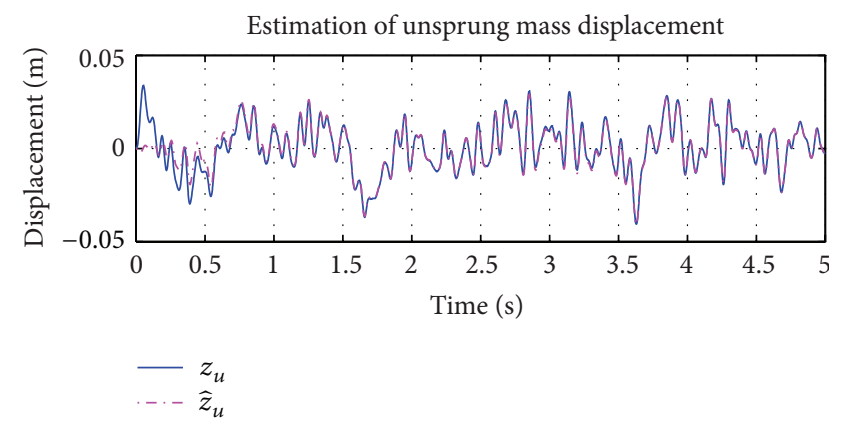

(d)

FIGURE 4: (a) Estimated unsprung mass velocity, (b) estimated sprung mass velocity, (c) estimated sprung mass displacement, (d) estimated unsprung mass displacement.

3.3. Estimation of Unknown Input: Road Excitation Profile. In the sliding mode, with the error $e_{1}$ converging to zero in finite time $\left(e_{1}=\dot{e}_{1}=0\right)$, the equivalent control [29] can be obtained from (18) as follows:

$$
v_{\mathrm{eq}}=-\Lambda\left(e_{1}, e_{z}, t\right)+E_{1} \zeta(t)
$$

As $e_{1} \rightarrow 0$ in finite time, and the nonlinearities satisfy Lipschitz assumptions, we have from (18) the following:

$$
\begin{array}{r}
\left\|\Lambda\left(e_{1}, e_{z}, t\right)\right\| \leq\left(\left\|A_{12}-L C_{2}\right\|+l_{\beta}\right)\|e(t)\| \longrightarrow 0 \\
(t \longrightarrow \infty) .
\end{array}
$$

The unknown road excitation profile when $t \rightarrow \infty$ can be thus obtained as

$$
\widehat{\zeta}(t)=E_{1}^{-1} K_{2} \int_{0}^{t} \operatorname{sign}\left(e_{1}\right) d t .
$$

Remark 8. The design of the adaptive STA observer and the NLO considered in this work can be easily extended for estimation of multiple unknown inputs. The general class of nonlinear systems that is similar to (6) is represented by

$$
\begin{aligned}
& \dot{x}=A x+B \Psi(x, u, t)+E f(t), \\
& y=C x,
\end{aligned}
$$

where $x \in M \subset \mathbb{R}^{n}, A \in \mathbb{R}^{n \times n}, C \in \mathbb{R}^{p \times n}, f(t)=$ $\left[f_{1}(t) \cdots f_{m}(t)\right] \in \mathbb{R}^{m}$, with $m<p \leq n$ are the unknown inputs/uncertainties under similar assumptions, general class of nonlinear systems is defined in (35); a linear transformation [10] can always be employed to obtain the required structure for the design of the observers.

\section{Results}

For the performance evaluation of the proposed observer, we select the following active suspension system parameters of a Ford Fiesta MK2 [30] vehicle: $m_{s}=216.75 \mathrm{~kg}, m_{u}=28.85 \mathrm{~kg}$, $k_{s}=21700 \mathrm{~N} / \mathrm{m}, b_{s}=1200 \mathrm{Ns} / \mathrm{m}$, and $k_{r}=184000 \mathrm{~N} / \mathrm{m}$. The nonlinear spring stiffness, $k_{s \mathrm{nl}}$, and damping constant, $b_{s \mathrm{nl}}$, values are taken as $10 \%$ of the original linear values $k_{s}$ and $b_{s}$, respectively. To design the adaptive STA, we choose the gains as $\kappa_{1}=500, \kappa_{2}=4, \kappa_{3}=30, \epsilon=0.3$, and $\alpha_{m}=4$. The initial conditions for the plant and the observer were chosen as $x(0)$ $=\left[\begin{array}{llll}0.1 & 0 & 0 & 0.01\end{array}\right]$ and $\widehat{x}(0)=\left[\begin{array}{llll}0 & 0 & 0 & 0\end{array}\right]$. The feedback gain for the subsystem (12) and the positive definite matrix, $Q$, satisfying (23) were computed as

$$
L=\left[\begin{array}{l}
6.0259 \\
7.2397 \\
3.3748
\end{array}\right], \quad Q=\left[\begin{array}{ccc}
0.0079 & -0.0031 & 0.0029 \\
-0.0031 & 0.0108 & -0.0020 \\
0.0029 & -0.0020 & 0.0111
\end{array}\right] \text {. }
$$

The Lipschitz constant for subsystem (12) was evaluated as $l_{\beta}=10.5651$. The road profile model [31] can be obtained as

$$
\dot{\zeta}(t)=-2 \pi n_{0} v \zeta(t)+2 \pi \sqrt{\sigma_{0} v} w_{0},
$$

where $v$ is the vehicle longitudinal velocity, $\sigma_{0}$ is the road roughness coefficient, $n_{0}$ is the reference space frequency, and $w_{0}$ is the Gaussian white noise. With changes in road roughness coefficient keeping the longitudinal velocity of motion fixed, different excitation profiles can be obtained. The increase in roughness coefficient leads to poorer road 


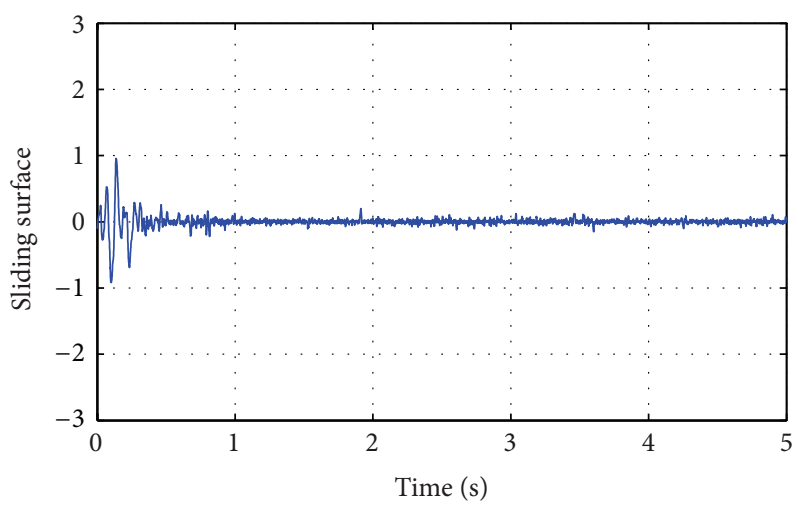

(a)

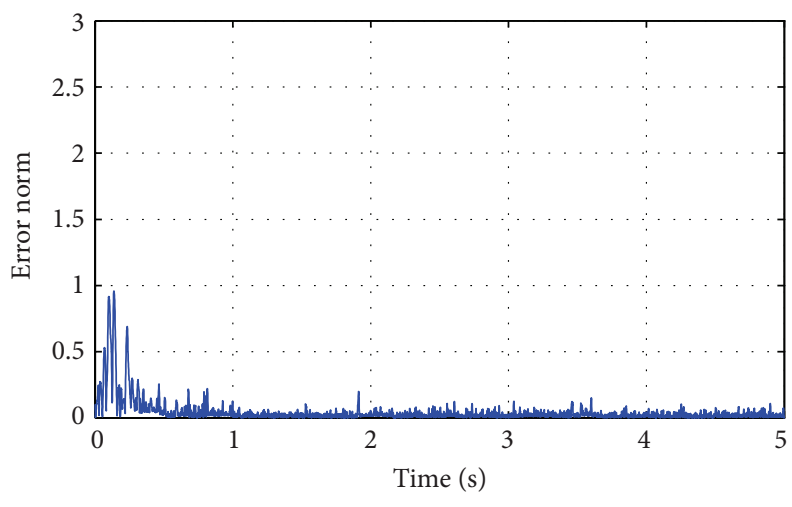

$\|e\|$

Figure 5: (a) Sliding surface $\left(e_{1}\right)$ and (b) Error norm $\|e\|$.

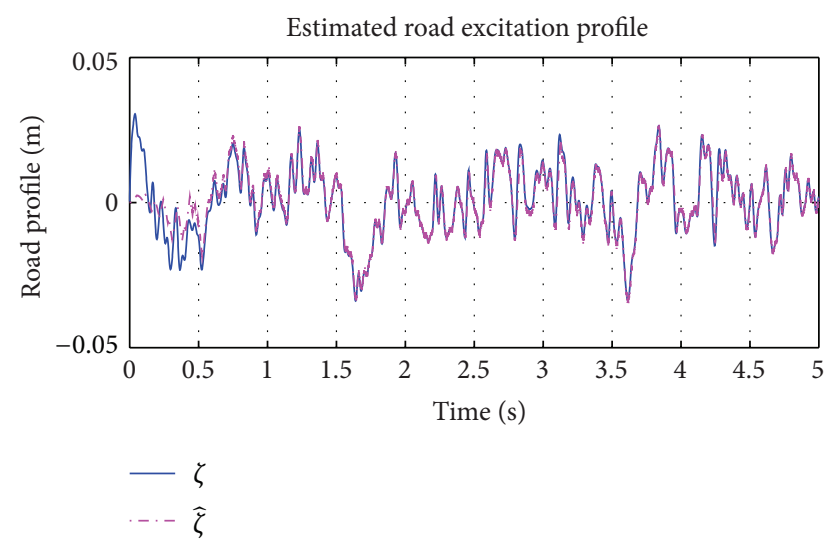

FIGURE 6: Estimation of road profile, $\zeta(t)$.

conditions. For simulation purposes, the class $\mathrm{C}$ road profile was considered as an unknown input. The vehicle was considered to be travelling at a speed of $30 \mathrm{Km} / \mathrm{hr}$ with road roughness coefficient being considered as shown in Table 1. The generated road profile is shown in Figure 3. The simulation results obtained for state estimation are shown in Figure 4 . The system dynamics are nonlinear and affected by the road profile, $\zeta(t)$, which is a function of white Gaussian noise. It can be deduced that the estimation of the states even under the effect of $\zeta(t)$ is good. The norm of the estimation error of the states is shown in Figure 5. In Figure 6, the unknown road excitation profile estimated with (34) is shown. A smooth estimation of the unknown road profile is obtained without any low-pass filtering.

\section{Conclusions}

In this work, an adaptive super-twisting observer was proposed for state and unknown input estimation for the active suspension system. The paper considered the nonlinear model of the active suspension system excited by the random road profile as an unknown input. Under the Lipschitz condition for the nonlinear functions, the convergence of the system errors is proven. The proposed adaptive supertwisting observer accurately estimates the road excitation profile for an average road without the use of low-pass filter.

\section{Conflict of Interests}

The authors declare that there is no conflict of interests regarding the publication of this paper.

\section{Acknowledgment}

This research was supported by the Basic Science Research Program through the National Research Foundation of Korea (NRF) funded by the Ministry of Education, Science and Technology (Grant no. 2011-0023999).

\section{References}

[1] D. Alexander, High-Performance Handling for Street or Track, Motor Books International, 1st edition, 2013.

[2] D. Knowles and J. Erjavec, Automotive Suspension and Steering Systems, Cengage Learning, 5th edition, 2002.

[3] R. Rajamani, Vehicle Dynamics and Control, Springer, New York, NY, USA, 2012.

[4] American Society of Testing and Materials, "Standard test method for measuring the longitudinal profile of traveled surfaces with an accelerometer established inertial profiling reference, ASTM E950," in Annual Book of ASTM Standards, vol. 4.03, 2004.

[5] American Society of Testing and Materials, "Standard test method for measuring pavement roughness using a profilograph," in Annual Book of ASTM Standards, vol. 4.03, 2008.

[6] D. Hugo, S. P. Heyns, R. J. Thompson, and A. T. Visser, "Condition-triggered maintenance for mine haul roads with reconstructed-vehicle-response to haul road defects," Journal of the Transportation Research Record, vol. 2, no. 1989, pp. 254-260, 2007. 
[7] M. Doumiati, A. Victorino, A. Charara, and D. Lechner, "Estimation of road profile for vehicle dynamics motion: experimental validation," in Proceedings of the American Control Conference (ACC '11), pp. 5237-5242, San Francisco, Calif, USA, July 2011.

[8] M. Yousefzadeh, S. Azadi, and A. Soltani, "Road profile estimation using neural network algorithm," Journal of Mechanical Science and Technology, vol. 24, no. 3, pp. 743-754, 2010.

[9] T. Yoshimura, A. Kume, M. Kurimoto, and J. Hino, "Construction of an active suspension system of a quarter car model using the concept of sliding mode control," Journal of Sound and Vibration, vol. 239, no. 2, pp. 187-199, 2001.

[10] C. Edwards, S. K. Spurgeon, and R. J. Patton, "Sliding mode observers for fault detection and isolation," Automatica, vol. 36, no. 4, pp. 541-553, 2000.

[11] X.-G. Yan and C. Edwards, "Nonlinear robust fault reconstruction and estimation using a sliding mode observer," Automatica, vol. 43, no. 9, pp. 1605-1614, 2007.

[12] R. K. Dixit and G. D. Buckner, "Sliding mode observation and control for semiactive vehicle suspensions," Vehicle System Dynamics, vol. 43, no. 2, pp. 83-105, 2005.

[13] K. C. Veluvolu, M. Defoort, and Y. C. Soh, "High-gain observer with sliding mode for nonlinear state estimation and fault reconstruction," Journal of Franklin Institute, 2013.

[14] K. C. Veluvolu, M. Y. Kim, and D. Lee, "Nonlinear sliding mode high-gain observers for fault estimation," International Journal of Systems Science, vol. 42, no. 7, pp. 1065-1074, 2011.

[15] K. C. Veluvolu and D. Lee, "Sliding mode high-gain observers for a class of uncertain nonlinear systems," Applied Mathematics Letters, vol. 24, no. 3, pp. 329-334, 2011.

[16] K. C. Veluvolu and Y. C. Soh, "Fault reconstruction and state estimation with sliding mode observers for Lipschitz non-linear systems," IET Control Theory and Applications, vol. 5, no. 11, pp. 1255-1263, 2011.

[17] K. C. Veluvolu and Y. C. Soh, "Multiple sliding mode observers and unknown input estimations for Lipschitz nonlinear systems," International Journal of Robust and Nonlinear Control, vol. 21, no. 11, pp. 1322-1340, 2011.

[18] L. Fridman, Y. Shtessel, C. Edwards, and X.-G. Yan, "Higherorder sliding-mode observer for state estimation and input reconstruction in nonlinear systems," International Journal of Robust and Nonlinear Control, vol. 18, no. 4-5, pp. 399-412, 2008.

[19] Y. Zhou, Y. C. Soh, and J. X. Shen, "High-gain observer with higher order sliding mode for state and unknown disturbance estimations," International Journal of Robust and Nonlinear Control, 2013.

[20] H. Rios, J. Davila, and L. Fridman, "High-order sliding mode observers for nonlinear autonomous switched systems with unknown inputs," Journal of the Franklin Institute, vol. 349, no. 10, pp. 2975-3002, 2012.

[21] A. Levant, "Sliding order and sliding accuracy in sliding mode control," International Journal of Control, vol. 58, no. 6, pp. 12471263, 1993.

[22] N. K. M’Sirdi, A. Rabhi, L. Fridman, J. Davila, and Y. Delanne, "Second order sliding mode observer for estimation of velocities, wheel sleep, radius and stiffness," in Proceedings of the American Control Conference, pp. 3316-3321, Minneapolis, Minn, USA, June 2006.

[23] H. Imine, Y. Delanne, and N. K. M'Sirdi, "Road profile input estimation in vehicle dynamics simulation," Vehicle System Dynamics, vol. 44, no. 4, pp. 285-303, 2006.
[24] H. Imine and V. Dolcemascolo, "Sliding mode observers to heavy vehicle vertical forces estimation," International Journal of Heavy Vehicle Systems, vol. 15, no. 1, pp. 53-64, 2008.

[25] Y. Shtessel, M. Taleb, and F. Plestan, "A novel adaptive-gain supertwisting sliding mode controller: methodology and application," Automatica, vol. 48, no. 5, pp. 759-769, 2012.

[26] U. Kiencke and L. Nielsen, Automotive Control Systems: For Engine, Driveline, and Vehicle, Springer, New York, NY, USA, 2005.

[27] R. Rajamani, “Observers for Lipschitz nonlinear systems," IEEE Transactions on Automatic Control, vol. 43, no. 3, pp. 397-401, 1998.

[28] R. Rajamani and Y. M. Cho, "Existence and design of observers for nonlinear systems: relation to distance to unobservability," International Journal of Control, vol. 69, no. 5, pp. 717-731, 1998.

[29] V. I. Utkin, Sliding Mode in Control and Optimization, Springer, New York, NY, USA, 1992.

[30] M. Jamei, M. Mahfouf, and D. Linkens, "A GA tuned fuzzy controller for a non-linear active suspension system," in Proceedings of the 7th UK Workshop on Fuzzy Systems, vol. 2, pp. 143-146, 2000.

[31] J. Cao, H. Liu, P. Li, and D. J. Brown, "State of the art in vehicle active suspension adaptive control systems based on intelligent methodologies," IEEE Transactions on Intelligent Transportation Systems, vol. 9, no. 3, pp. 392-405, 2008. 

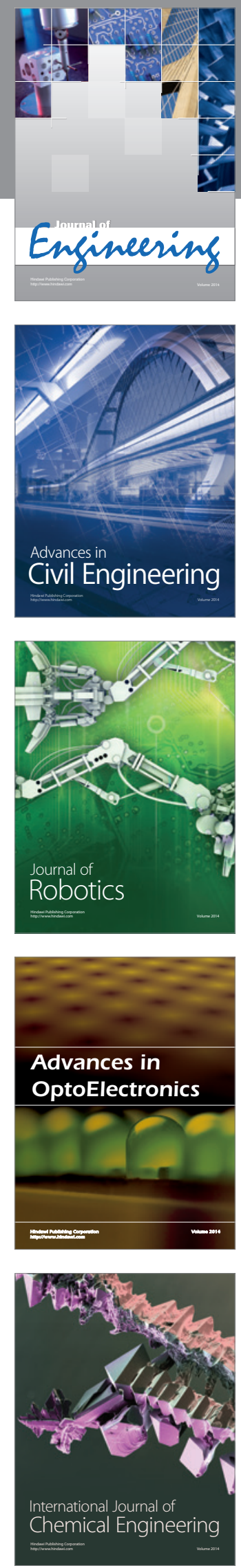

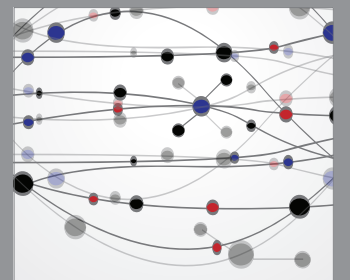

The Scientific World Journal
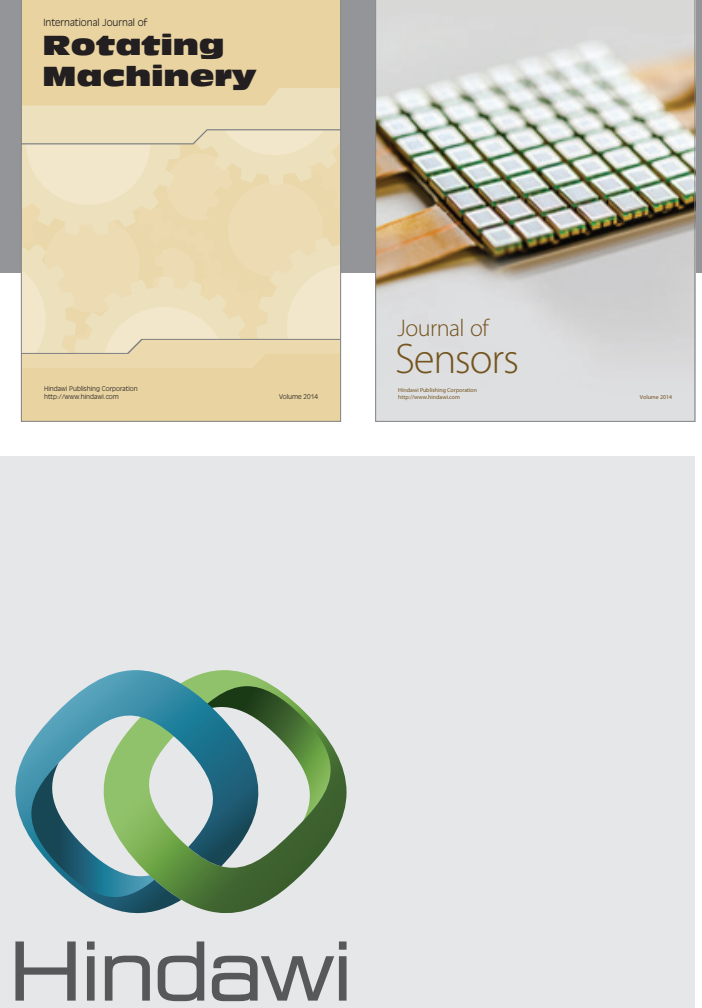

Submit your manuscripts at http://www.hindawi.com
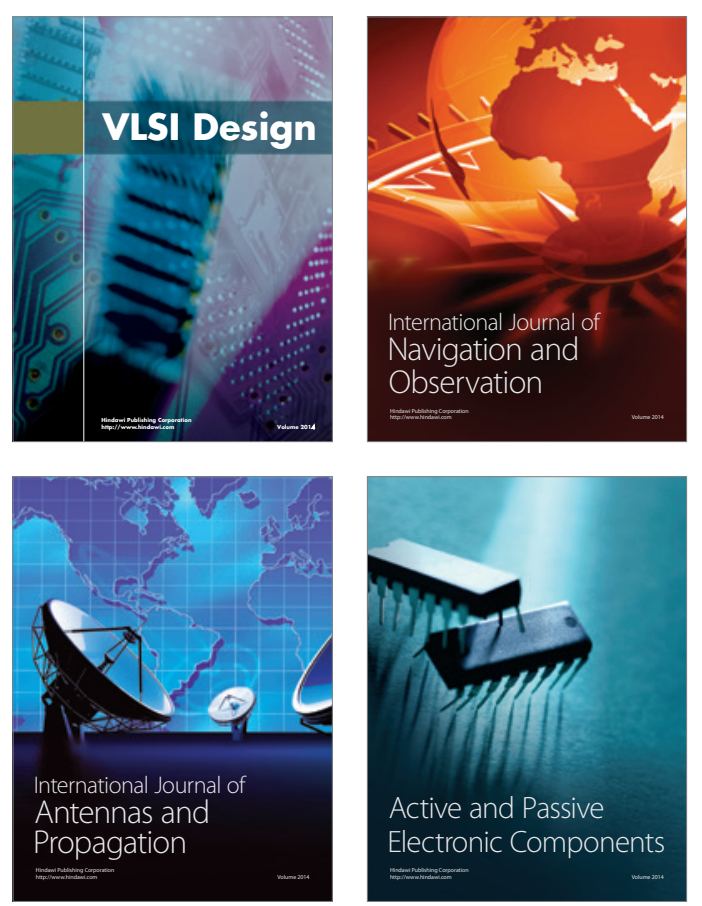
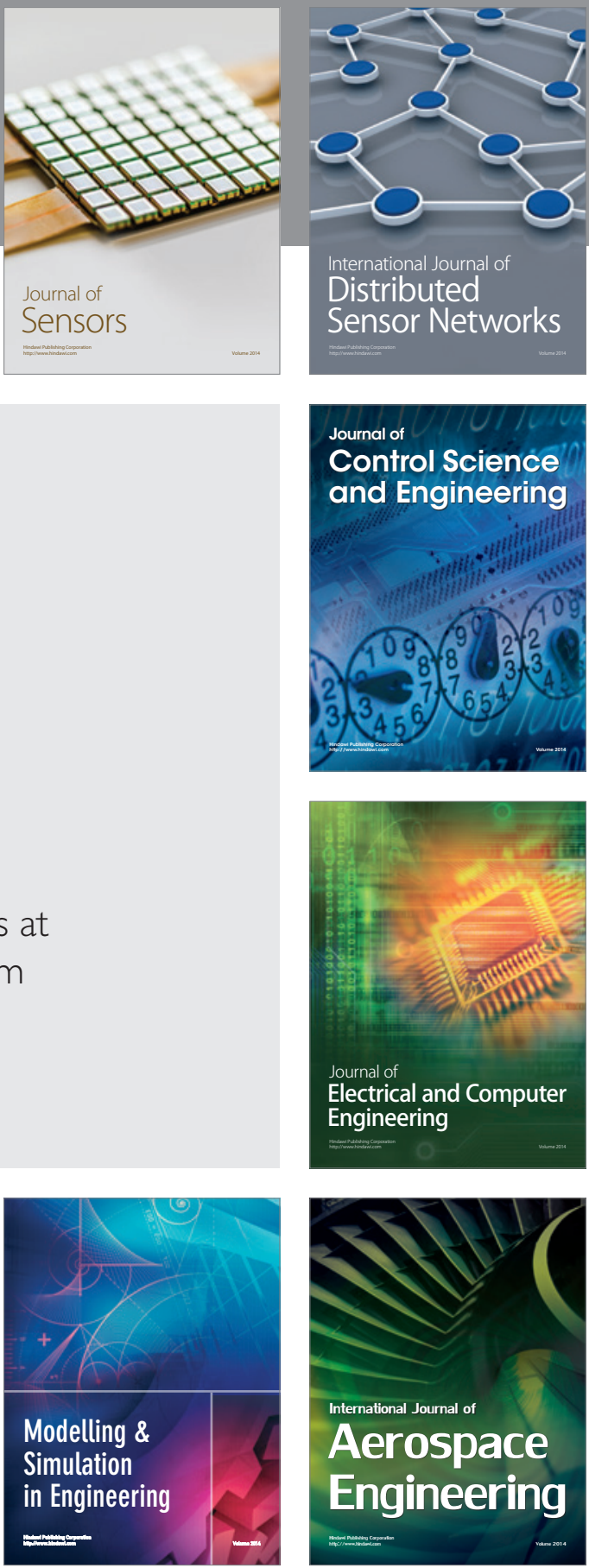

Journal of

Control Science

and Engineering
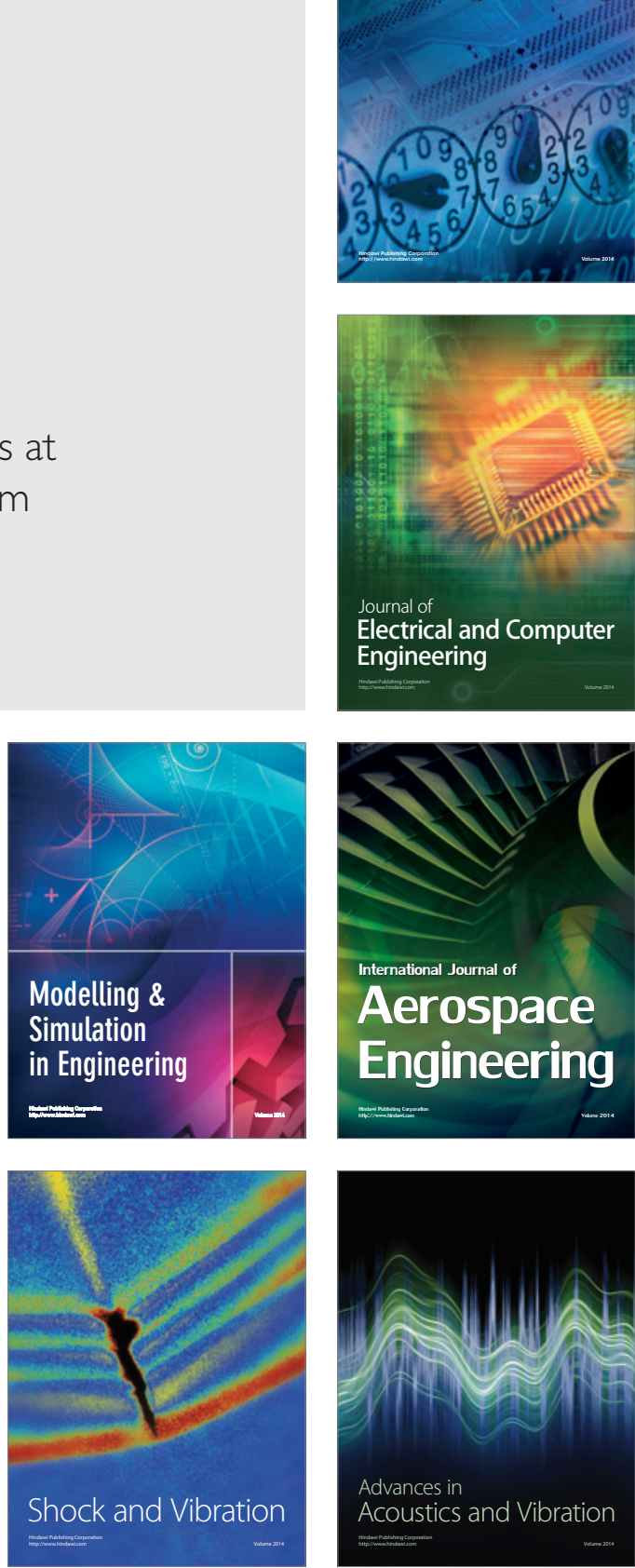